\title{
Multistatic SAR Imaging: Comparison of Simulation Results and Experimental Data
}

\author{
T. Kraus*, M. Bachmann*, L. Heiderich ${ }^{\dagger}$, G. Krieger*, A. Moreira* \\ *Microwaves and Radar Institute, German Aerospace Center(DLR), Oberpfaffenhofen, Germany,t.kraus@dlr.de \\ ${ }^{+}$University of Applied Sciences, Munich, Germany
}

Keywords: Synthetic aperture radar (SAR), multistatic SAR, high-resolution wide-swath SAR, azimuth ambiguities, TerraSAR-X, TanDEM-X.

\begin{abstract}
Synthetic aperture radar (SAR) systems in a multistatic configuration are a promising candidate for future Earth observation and reconnaissance radar systems. They overcome the sampling constraints inherent to single-channel SAR systems. Thus, a multistatic SAR system enables the acquisition of high-resolution images while maintaining wideswath coverage. Employing several small satellites instead of a single large one, a cost-efficient system with graceful degradation characteristics can be envisaged. Additionally, such a constellation or swarm of sensors offers interferometric and tomographic capabilities, which a singlesatellite system is not able to provide. This paper shows results of multistatic experiments obtained with TerraSAR-X and TanDEM-X and compares these results with theoretical simulations. The key parameters analyzed are the Doppler spectrum and the azimuth ambiguity suppression.
\end{abstract}

\section{Introduction}

The capabilities of classical single-channel SAR systems regarding wide-swath imaging with high azimuth resolution are inherently limited by contradicting pulse repetition frequency (PRF) requirements. Wide swathes require large echo windows and therefore a small PRF. However, the wide Doppler spectrum of high-resolution images demands a high PRF in order to adequately sample the signal respecting the Nyquist criterion. Violations of the sampling criterion lead to rising azimuth ambiguities in the image and reduce the interpretability of the data products. Using multiple phase centers offers the ability to overcome the sampling constraints and acquire high-resolution wide-swath images [1], [2]. Spaceborne experiments with two channels of TerraSAR-X [3] operated in the "dual receive antenna" (DRA) [4] mode and the TerraSAR-X and TanDEM-X formation flying system [5] have been reported in the literature. Also a four channel experiment with TerraSAR-X and TanDEM-X jointly operating in DRA mode has been conducted [6]. These experimental datasets were acquired during a dedicated science phase [7] which was conducted after the completion of the primary mission goal of TanDEM-X, the acquisition of a global digital elevation model (DEM) with outstanding accuracy [8]. During the science phase the geometry of the formation flying interferometer was adjusted several times in order to serve the demands of different scientific applications. Due to the suitability of the formation during winter 2014 and the flexibility of the TanDEM-X instrument commanding an experiment like the one described in [6] was possible. Based on these results the paper at hand compares actual multichannel SAR signal reconstruction data with simulation results provided by a dedicated simulation framework. The goal of the comparison is to validate the simulation results against actual acquired data. Furthermore, the simulation framework is highly flexible in terms of the choice of system parameters and the underlying geometries. Therefore, it can serve as a tool for the design and performance assessment of future multistatic SAR missions.

The paper is structured as follows. Section 2 describes the experimental dataset. In section 3 the simulation framework is shown, followed by a comparison of experimental and simulated data in section 4 . The paper is summarized in section 5 .

\section{Experimental Data}

For the experiment dedicated data takes in stripmap mode have been acquired. The commanding was adjusted to PRFs lower than nominal in order to provoke azimuth ambiguities in the single-channel SAR data. The goal is to reduce these ambiguities by azimuth signal reconstruction. The actual scene was chosen to contain a high contrast region, e.g., a land-sea transition, where azimuth ambiguities are easily recognized as shown in Figure 1 on the left. The right image shows a two-channel reconstructed image with lower ambiguity level.
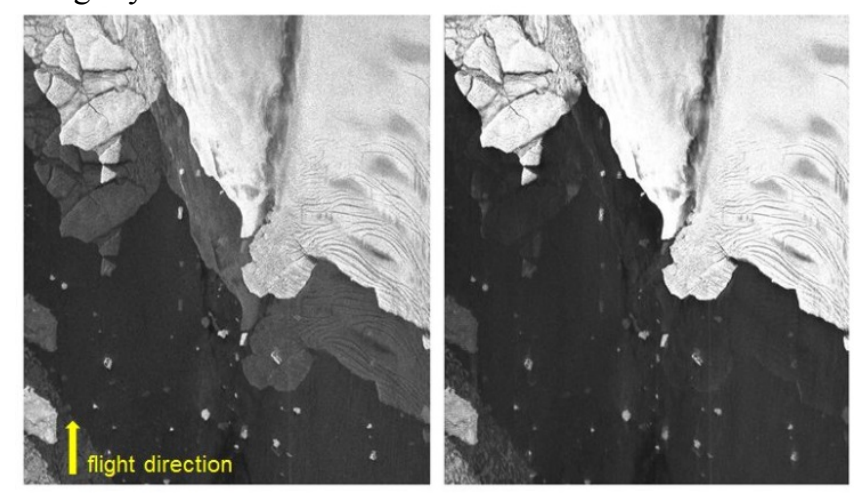

Figure 1: Single-channel acquisition of TerraSAR-X showing prominent azimuth ambiguities (left) and the reconstructed image with lower ambiguity level based on TerraSAR-X and TanDEM-X (right). 


\subsection{Satellite Formation}

In December 2014 the satellites were in a pursuit monostatic formation. Both radars acquired the same scene independently with an along-track separation of about ten seconds, using identical PRFs. A sketch of the acquisition scenario is given in Figure 2. Each satellite is symbolized by a parallelogram with two antenna halves oriented in flight direction. The blue solid circles represent the effective phase centers of the four channels. The shown along-track baselines $\Delta x_{n}$ are referenced to the fore channel of TerraSAR-X. The sample spacing for both channels of one platform is given by the geometry of the SAR antenna. The mutual spacing of the spatial sampling between the satellites could not be controlled since it depends on the start of the acquisitions which is independently triggered by the on-board start time correction mechanism of the instruments. At a PRF of $2000 \mathrm{~Hz}$ the sample spacing is about $3.8 \mathrm{~m}$ which is well below the accuracy of the start time correction mechanism. Due to the satellite formation, small or vanishing cross-track baselines could only be acquired very south, close to Antarctica.



Figure 2: Sketch of the acquisition geometry of the experiment showing both satellites TerraSAR-X (TSX) and TanDEM-X (TDX). The red solid and the blue nonsolid circles represent the transmit and receive antenna phase centers, respectively. The blue solid circles are the effective phase centers on each platform employing the DRA mode. The along-track baselines $\Delta x_{n}$ are shown relative to the leading edge phase center of TerraSAR-X, called the fore channel.

\subsection{Dual Receive Antenna Mode}

During nominal operations of TerraSAR-X the receive signals of both halves of the antenna are added using a hybrid coupler and recorded as the sum channel. This channel experiences the full azimuth aperture of $4.8 \mathrm{~m}$ in transmit and receive. In Figure 2 the respective phase centers are shown as red solid circles. Using the DRA mode means to activate the redundant receiver chain and to record also the signal on the difference port of the hybrid coupler. By using a calibration and reconstruction algorithm it is possible to get the so called fore and aft signals of both antenna halves [3]. These channels are characterized by a wider Doppler spectrum than the sum channel, since the azimuth length of the receiving subapertures is only half the full aperture.

The multichannel data can be evaluated by the approach which is sketched in [6]. Depending, whether only both sum channels of TerraSAR-X and TanDEM-X are used, or the fore and aft channels are used on both satellites, the number of channels for the multichannel azimuth reconstruction can range from two to four.

\section{Simulation Framework}

The simulation framework is implemented with very flexible input parameters. Its core part is a point target simulator. A block diagram is shown in Figure 3. The inputs are on the one hand the system parameters like the number of apertures, the aperture sizes, the PRF, the signal-to-noise ratio (SNR), and other processing parameters. On the other hand, the geometry of the data acquisition is parametrized by the sensors trajectories, velocities and attitudes. These input parameters are either chosen to match with a certain real acquisition to compare the simulated performance results with measured ones, or they are set to predefined values in order to estimate the performance of a possible future multistatic SAR mission. The core part of the simulation framework consists of the generation of the signals and the implementation of the azimuth signal reconstruction as described in [1] or [9]. In a subsequent step the reconstructed signal is analyzed regarding different parameters, e.g., the azimuth ambiguity-to-signal ratio (AASR) or the shape of the Doppler spectrum.

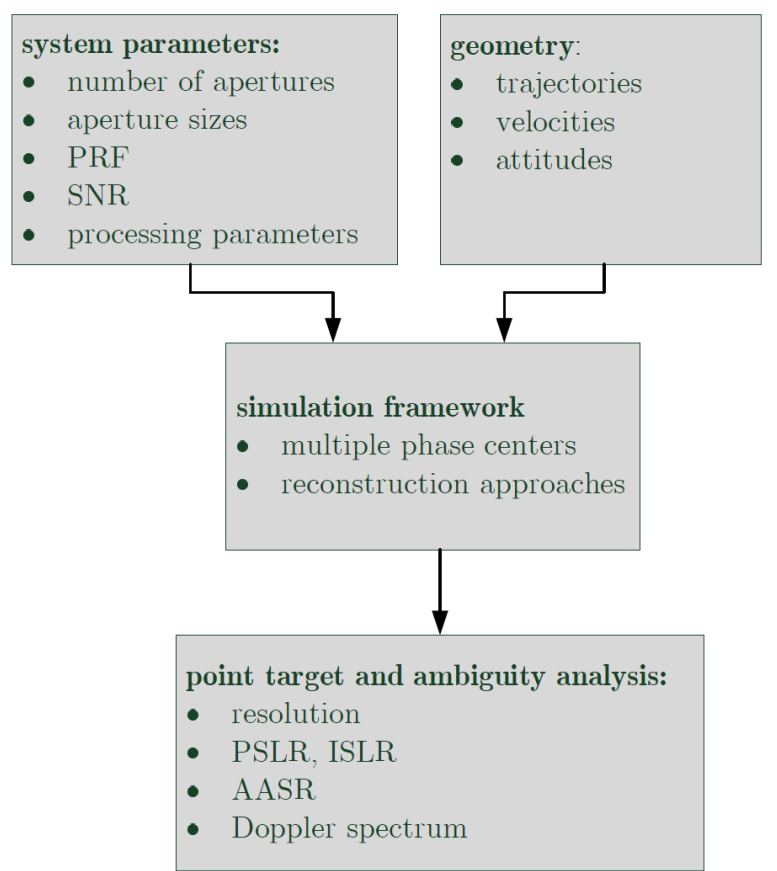

Figure 3: Block diagram of the framework used to simulate the azimuth signal reconstruction. 


\section{Comparison of Experimental and Simulation Results}

The multichannel SAR data of the acquisitions is processed in accordance with the evaluation approach presented in [6]. The Doppler spectrum as well as the azimuth ambiguity performance is evaluated.

\subsection{Two-Channel Reconstruction}

By evaluating a high-backscatter area of the scene, a Doppler spectrum of the reconstructed signal can be derived. The spectrum of a two-channel reconstructed signal is shown in blue in Figure 4. The red curve is derived using the simulation framework. The input parameters of the simulation like the PRF, the separation of the apertures, and the processing parameters are set in accordance with the values of the actual acquisition. Since the incidence angle of the acquisition is approximately $30^{\circ}$ and the evaluated region is covered with snow assuming a SNR of $20 \mathrm{~dB}$ seems to be realistic. Taking this as the single channel SNR, the red Doppler spectrum can be calculated. Both spectra fit very well, indicating a successful reconstruction.

For comparison the azimuth reference antenna pattern is shown in green. It can serve as rough hint what the reconstructed Doppler spectrum should look like. However, it does neither take into account residual ambiguities from outside the reconstructed azimuth frequency band, nor does it consider noise. Therefore, the simulated spectrum proofs to be very valuable when evaluating multichannel reconstructed SAR data or estimating the performance of future multistatic SAR systems.

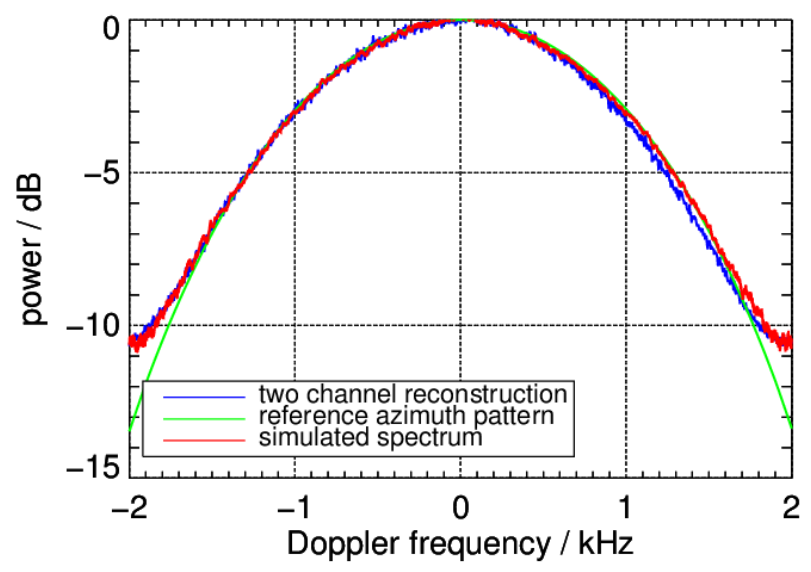

Figure 4: The reconstructed Doppler spectrum of both sum channels over a bandwidth of $4 \mathrm{kHz}$ in blue. The simulated spectrum after the two-channel reconstruction is shown in red. For comparison the two-way azimuth antenna pattern is shown in green.

In order to evaluate the AASR performance of the actual images an azimuth profile along a high contrast region can be analyzed as shown in Figure 5 for the two-channel reconstructed image. The normalized power profile of the single channel TerraSAR-X image is shown in blue in Figure 6 . The green curve represents the power profile of the two-channel reconstructed data. For comparability reasons both images have been processed to the same azimuth resolution. Therefore, a SNR gain of $3 \mathrm{~dB}$ is visible in the azimuth range between 0 and $5.8 \mathrm{~km}$ for the two-channel data. In this area the backscatter of the sea is very low and the received signal is dominated by the noise power. The ambiguity power is clearly visible between 5.8 and $8.2 \mathrm{~km}$, especially for the single channel profile. The distance of about $2.4 \mathrm{~km}$ between the actual target area and its ambiguity coincides with the predicted position. The dominant ambiguity power originates form the area between 8.2 and $10.6 \mathrm{~km}$. Comparing the power levels, an ambiguity suppression of about $-8 \mathrm{~dB}$ for the single channel profile and of about $-14 \mathrm{~dB}$ for the two-channel reconstructed profile is visible.

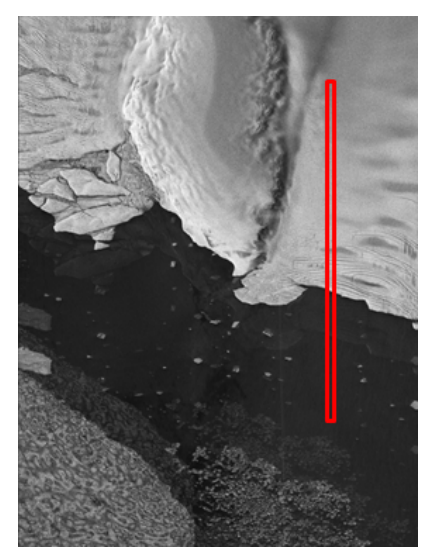

Figure 5: Zoom into the two-channel reconstructed image. The region of the azimuth profile which is evaluated to derive azimuth ambiguity ratios is highlighted in red.

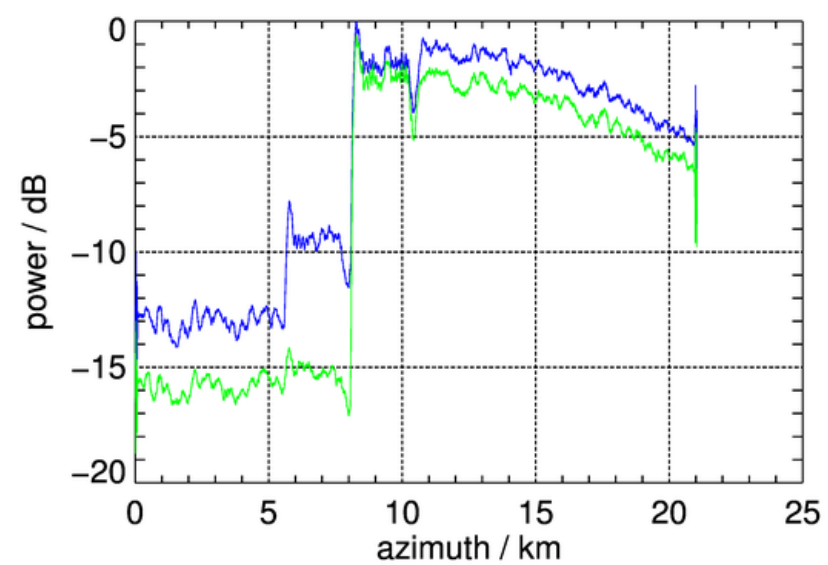

Figure 6: Azimuth profiles along the sea-land transition (red rectangle in Figure 5) for the single-channel TerraSAR-X dataset in blue and for the reconstructed dataset based on TerraSAR-X and TanDEM-X date in green.

In Figure 7 the simulated AASR for an acquisition scenario similar to the actual experimental acquisitions is shown as a function of PRF. The blue curve represents the AASR of a single channel. The red curve corresponds to the AASR after two-channel reconstruction. As expected the AASR performance of the single channel data is improving as the PRF increases. The AASR of the reconstructed signal 
however worsens again for PRFs larger than $2.25 \mathrm{kHz}$. The reason for this behaviour is the spatial sample distribution [1]. For the given geometry a PRF of about $2.2 \mathrm{kHz}$ leads to a uniform sampling of the received signals. Increasing the PRF further increases the non-uniformity of the sampling which compensates the benefit of a larger PRF and even further deteriorates the AASR performance. The PRF of the actual acquisition was $2 \mathrm{kHz}$ leading to a sampling uniformity of 0.6 for a scale between 0.0 and 2.0 (uniform sampling for 1.0). This results in a theoretical AASR performance of about $-15 \mathrm{~dB}$ for the reconstructed signal. The single channel achieves about $-5 \mathrm{~dB}$ AASR. In order to compare the simulated AASR values with the results obtained from Figure 6 the simulated values have to be adjusted by $-3 \mathrm{~dB}$. This is necessary as the simulation results consider ambiguities in both azimuth directions whereas the azimuth profiles are dominated by a single sided ambiguity, i.e., the strong backscatter region of the land side. The simulation levels for a comparison are thus $-8 \mathrm{~dB}$ for the single channel and $-18 \mathrm{~dB}$ for the reconstructed data. The single channel AASR value agrees very well with the actual value derived from the azimuth profile. However, for the reconstructed signal the simulation predicts an AASR of $-18 \mathrm{~dB}$ but the image shows $-14 \mathrm{~dB}$. This deviation might by caused by effects not jet considered within the simulation framework or by inaccuracies during the multi-channel reconstruction of the experimental data. Additionally, the residual ambiguity in Figure 6 is very close to the noise floor which complicates an assessment of lower ambiguity levels.

\subsection{Four-Channel Reconstruction}

The same AASR evaluation approach using azimuth profiles as used for the two-channel data was employed to assess the quality of four-channel reconstruction results. Since the reconstructed azimuth bandwidth is larger in the four-channel case, the images have been processed to a finer resolution. In Figure 8 the profiles of the two-channel reconstruction are shown in blue and the four-channel result is depicted in green.

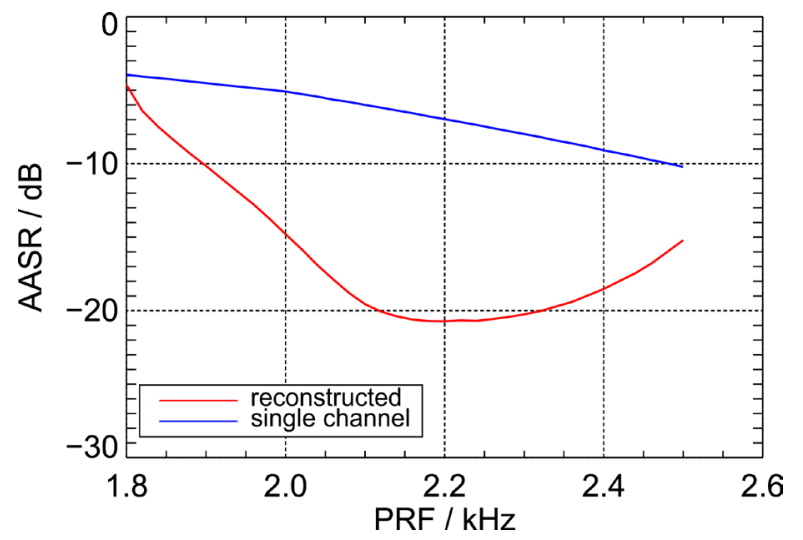

Figure 7: Simulated AASR performance for the geometry and system parameters equivalent to the experimental satellite acquisitions as a function of the PRF. The imaging PRF of the actual acquisition was $2 \mathrm{kHz}$.



Figure 8: Azimuth profiles along the sea-land transition for two-channel (blue) and the four-channel reconstructed datasets.

Comparing the tow-channel reconstruction result of Figure 6 and Figure 8 an approximately $3 \mathrm{~dB}$ higher ambiguity level is recognized. This is mainly due to the doubled azimuth processing bandwidth which is necessary to achieve the finer azimuth resolution. The four-channel reconstruction delivers an improved ambiguity performance of about $-13 \mathrm{~dB}$. From simulations however, an AASR level of $-16 \mathrm{~dB}$ is expected. The reasons for this deviation are the same as for the twochannel example.

Nevertheless, the parallel evaluation of actual experimental data and a simulation scenario tailored to closely mirror the actual acquisition geometry and parameters proved to be very helpful. On the one hand, in the simulation effects can be isolated and their influence can be evaluated over a wide parameter range. Having a real world example on the other hand, is helpful to identify challenges and to assess the applicability of methods for actual data.

\section{Summary}

This paper describes a simulation framework for the performance assessment of multichannel SAR systems. Comparisons of actual TerraSAR-X and TanDEM-X spaceborne acquisition results and simulations are shown. The simulation results are in good accordance with the evaluated experimental data. Some differences still exist. However, to identify such differences was the goal of the assessment approach in order to identify challenges and gain insight. These deviations are subject to further investigations. Further extensions to the framework focusing on different aspects of multichannel SAR systems like a more accurate compensation of the topographic phase or the time variance of the baselines are on-going.

\section{Acknowledgements}

The TanDEM-X data was acquired under the scientific proposal braeutig_OTHER0103. For the topography correction TanDEM-X DEM data was used which was ordered via the scientific proposal DEM_OTHER1279. 


\section{References}

[1] G. Krieger, N. Gebert, and A. Moreira, "Unambiguous SAR signal reconstruction from nonuniform displaced phase center sampling," IEEE Geosci. Remote Sens. Lett., vol. 1, no. 4, pp. 260-264, 2004.

[2] N. Gebert, G. Krieger, and A. Moreira, "Digital beamforming on receive: Techniques and optimization strategies for high-resolution wide-swath SAR imaging," IEEE Trans. Aerosp. Electron. Syst., vol. 45, no. 2, pp. 564-592, 2009.

[3] M. Gabele, B. Bräutigam, D. Schulze, U. Steinbrecher, N. Tous-Ramon, and M. Younis, "Fore and aft channel reconstruction in the TerraSAR-X dual receive antenna mode," IEEE Trans. Geosci. Remote Sens., vol. 48, no. 2, pp. 795-806, 2010.

[4] J.-H. Kim, M. Younis, P. Prats-Iraola, M. Gabele, and G. Krieger, "First spaceborne demonstration of digital beamforming for azimuth ambiguity suppression," IEEE Trans. Geosci. Remote Sens., vol. 51, no. 1, pp. 579590, 2013.

[5] M. Rodriguez-Cassola, P. Prats-Iraola, U. Steinbrecher, D. Schulze, G. Krieger, A. Reigber, and A. Moreira, "Cross-platform spaceborne SAR imaging: Demonstration using TanDEM-X," in Geoscience and Remote Sensing Symposium (IGARSS), 2013 IEEE International, 2013, pp. 2962-2965.

[6] T. Kraus, B. Bräutigam, M. Bachmann, and G. Krieger, "Multistatic SAR Imaging: First Results of a Four Phase Center Experiment with TerraSAR-X and TanDEM-X", in European Conference on Synthetic Aperture Radar (EUSAR), Hamburg, Germany, 2016.

[7] I. Hajnsek and T. E. Busche, "Announcement of opportunity: TanDEM-X science phase," German Aerospace Center (DLR), TD-PD-PL-0032 1.0, 2014.

[8] G. Krieger, A. Moreira, H. Fiedler, I. Hajnsek, M. Werner, M. Younis, and M. Zink, "TanDEM-X: A satellite formation for high-resolution SAR interferometry," IEEE Trans. Geosci. Remote Sens., vol. 45, no. 11, pp. 3317-3341, 2007.

[9] D. Cerutti-Maori, I. Sikaneta, J. Klare, and C. Gierull, "MIMO SAR processing for multichannel highresolution wide-swath radars," IEEE Trans. Geosci. Remote Sens., vol. 52, no. 8, pp.5034-5055, 2014. 